Introduction/Background Malignant sex cord stroma cell tumours (SCST) account for less than eight percent of ovarian malignancies. The Arbeitsgemeinschaft fuer Gynaekologische Onkologie (AGO) has established a clinicopathological (Current Ovarian geRm cell and SEx cord stromal Tumour Treatment strategies, CORSETT) database for a better documentation and understanding of this rare disease. Here, we present the first clinicopathological descriptive analysis for patients with independently confirmed SCST from the CORSETT database.

Methodology 20 German centres entered mixed retro- and prospective data of SCST patients with tumour specimens available treated between 2000 to 2014 into the CORSETT database. An independent CORSETT pathology reference panel checked the primary histological diagnosis.

We conducted a descriptive analysis of the treatment strategies and created Kaplan-Meier curves and cox regression analyses for the survival analysis.

Results The reference pathology panel diagnosed 143 patients with granulosa cell (GCT, FIGO stage $\mathrm{I}=120,87.0 \%$ ) and 14 patients with Sertoli-Leydig cell (SLCT, FIGO stage I = $11,91.7 \%$ ) tumours (others $=5$ ). The median age of patients with GCT was 57.6 years (SLCT: 47.2 years). 87 of GCT $(61.7 \%)$ and eight SLCT $(57.1 \%)$ patients were treated with laparoscopy and the tumour ruptured intraoperatively in $22 \%$ (SLCT: $7.7 \%)$ of the cases. 57 GCT (45\%) and eight SLCT $(57 \%)$ patients received fertility-sparing surgery. 19 of GCT $(15 \%)$ and two SLCT $(15.4 \%)$ patients received adjuvant chemotherapy. 59 of GCT (45\%) and two of SLCT (14.3\%) patients experienced a disease recurrence. The median progression-free survival (PFS) for all SCST patients was 80.4 months, (overall survival not reached). Advanced FIGO stage was associated with decreased PFS ( $p<0.05$ ).

Adjuvant chemotherapy had no statistically significant beneficial effect on PFS (all regimens $\mathrm{p}>0.05$ ).

Conclusion In this analysis, almost every fourth SCST patient treated surgically experienced an intraoperative cyst rupture that had however no impact on disease recurrence. One in five SCST patient received adjuvant chemotherapy that had no PFS improvement.

Disclosures

\section{TARGETING AKT AND DNA-PK AS A THERAPEUTIC STRATEGY IN PLATINUM RESISTANT HIGH-GRADE SEROUS OVARIAN CANCER}

${ }^{1}$ Natasha Rinne, 'Shengnan Yuan, ${ }^{1}$ Christina Fotopoulou, ${ }^{2}$ Hani Gabra, ${ }^{1}$ Paula Cunnea. ${ }^{1}$ Imperial College London; Division of Cancer, Department of Surgery and Cancer; ${ }^{2}$ Bergenbio, Magdalen Centre; Robert Robinson Avenue, The Oxford Science Park

\subsection{6/ijgc-2020-ESG0.112}

Introduction/Background High-grade serous ovarian cancer (HGSOC) is the most lethal form of gynaecological malignancy. Despite initial sensitivity to platinum chemotherapy, the majority of patients develop resistance to treatment and eventually die. Current treatment options for platinum-resistant patients are limited.

The role of the PI3K/AKT/mTOR pathway has been described in chemo-resistant HGSOC, in particular through activation of AKT by DNA-PK in response to platinum treatment. As increasing numbers of AKT and DNA-PK inhibitors advance to clinical trials, determining mechanism of action and efficacy is crucial.
This project aims to evaluate inhibition of AKT or DNA$\mathrm{PK}$ as a therapeutic strategy to target platinum resistance in HGSOC, and identify proteomic signatures confirming mechanism of action and target inhibition.

Methodology A panel of seven AKT and DNA-PK inhibitors were tested in combination with cisplatin chemotherapy in immortalised HGSOC cell lines and primary tumour cells cultured from HGSOC tumour/ascites samples. Clonogenic assays were performed to establish effect of inhibitor treatment in combination with cisplatin chemotherapy on the ability of cells to form colonies. Isobologram assays were performed to establish synergy/antagonism between inhibitors and cisplatin chemotherapy. Proteomic Reverse Phase Protein Array (RPPA) was performed to determine the mechanism of action of inhibitors, and results were confirmed with immunoblotting.

Results Treatment with AKT or DNA-PK inhibitors in combination with cisplatin led to significantly enhanced apoptotic responses in immortalised platinum-resistant HGSOC cell lines $(n=5)$, and in primary cells derived from ascites or tumour $(\mathrm{n}=4, \mathrm{p}<0.01, \mathrm{p}<0.05)$, compared to cisplatin treatment alone. In platinum-resistant HGSOC cell lines, fewer cell colonies were observed with increasing concentrations of AKT or DNA-PK inhibitors in combination with cisplatin $(n=3)$ in comparison with cisplatin alone. Varying synergistic effects were observed across the panel of inhibitors when combined with cisplatin; Uprosertib (AKT inhibitor) in particular displayed strong synergy with cisplatin (Loewe analysis). Proteomic analysis of inhibitor treatment in HGSOC platinumresistant cells demonstrated the mechanism of action of Uprosertib in targeting the PI3K/AKT pathway.

Conclusion In platinum-resistant HGSOC cells, AKT or DNAPK inhibition functioned synergistically with cisplatin and reduced cell growth and proliferation. In both immortalised and primary HGSOC cell lines tested, AKT or DNA-PK inhibition significantly enhanced the apoptotic response to cisplatin demonstrating the efficacy of AKT or DNA-PK as potential therapeutic targets in chemoresistant HGSOC. By improving patient response to treatment, AKT and DNA-PK inhibitors could expand the therapeutic options for patients with platinum-resistant HGSOC, improving overall survival.

Disclosures CF: advisory boards and honoraria from Roche, Tesaro, Sequana, Olympus, Astra Zeneca. Other authors have no conflict of interest.

\section{TREATMENT STRATEGIES AND SURVIVAL OF WOMEN WITH MALIGNANT OVARIAN GERM CELL TUMOURS - AN ANALYSIS OF THE AGO-CORSETT DATABASE}

${ }^{1}$ Annette Hasenburg, ${ }^{2}$ Helmuth Plett, ${ }^{3}$ Philipp Harter, ${ }^{4}$ Stefan Kommoss, ${ }^{5}$ Jaqueline Keul, ${ }^{6}$ Eva Roser, ${ }^{7}$ Bastian Czogalla, ${ }^{8}$ Michaela Bossart, ${ }^{9}$ Theresa Link, ${ }^{10}$ Maximilian Klar. ${ }^{1}$ Dept Gynecology and Obstetrics; University Center Mainz; Klinik und Poliklinik für Geburtshilfe und Frauengesundheit; ${ }^{2}$ Klinikum Essen Mitte; ${ }^{3}$ Klinikum Essen Mitte; Gynaecology and Gynaecological Oncology; ${ }^{4}$ Universitätsklinikum Tübingen; Universitätsfrauenklinik; University Hospital Tuebingen; ${ }^{5}$ University Hospital Tuebingen; Universitätsfrauenklinik; ${ }^{6}$ Charite Berlin; ${ }^{7}$ MUU Munich; Obstetrics and Gynaecology; ${ }^{8}$ Universitätsklinikum Freiburg; University of Freiburg; Obstetrics and Gynaecology; ${ }^{9}$ Uniklinik Dresden; Gynäkologie und Geburtshilfe; Obstetrics and Gynaecology; ${ }^{10}$ University of Freiburg

\subsection{6/ijgc-2020-ESGO.113}

Introduction/Background Malignant ovarian germ cell tumours (OGCT) account for about five percent of all ovarian malignancies in Western countries. The Arbeitsgemeinschaft fuer 